\title{
USO DO NITRATO DE POTÁSSIO NO TRATAMENTO DA HIPERSENSIBILIDADE DENTINÁRIA: UMA REVISÃO DE LITERATURA
}

\section{USE OF POTASSIUM NITRATE IN THE TREATMENT OF DENTIN HYPERSENSITIVITY: A LITERATURE REVIEW}

Héberte de Santana Arruda ${ }^{1 *}$ (D), Mariana Alves Lemos $^{2}$ (D), Marcella Lydia Parente Mecozzi ${ }^{2}$ (D), Zilda Betânia Barbosa Medeiros de Farias $^{1}$ (D), Amanda Maciel do Prado ${ }^{1}$ (D), Marcos Antonio Japiassú Resende Montes ${ }^{1}$ (i)

\author{
${ }^{1}$ Universidade de Pernambuco, Camaragibe, PE, Brasil. \\ ${ }^{2}$ Instituto de Medicina Integral Professor Fernando Figueira, Recife, PE, Brasil. \\ *hebertearruda@gmail.com
}

\section{RESUMO}

Este trabalho teve como objetivo realizar uma revisão de literatura sobre o tratamento da hipersensibilidade dentinária com agentes dessensibilizantes a base de Nitrato de potássio. Foi realizado levantamento bibliográfico do período de 2003 a 2019 nas bases de dados PubMed e Scielo. Foram utilizadas as palavras chaves em português "Desmineralização" "Hipersensibilidade da Dentina" e "Nitrato de Potássio" e as correspondentes em inglês, "Demineralization" "Dentin Sensitivity" e "Potassium Nitrate". A literatura apontou que o nitrato de potássio atua aumentando a concentração de íons potássio na extremidade interna dos túbulos dentinários em nível suficiente para inativar às terminações nervosas da polpa. Isto provocou a despolarização das fibras nervosas, apresentando ação mais rápida e duradoura quando comparado a outros agentes dessensibilizantes. Dentifrícios a base de $\mathrm{KNO}_{3}$ sozinho ou combinados com outros componentes mostraram-se capazes de tratar de maneira eficaz a hipersensibilidade dentinária. As formas de incorporação do nitrato de potássio mais frequentes são através de dentrifícios e colutórios bucais onde a posologia que mais apresentou resultados satisfatórios para o uso dos mesmos no tratamento da hipersensibilidade dentinária foi a associação da escovação e enxágue, respectivamente, duas vezes ao dia durante dois minutos. Todos os agentes dessensibilizantes a base de nitrato de potássio analisados no presente estudo foram capazes de tratar de maneira eficaz a hipersensibilidade dentinária.

Palavras-chave: Desmineralização. Hipersensibilidade da Dentina. Nitrato de Potássio.

\section{ABSTRACT}

The aim of this study was to conduct a review of the literature on the treatment of dentin hypersensitivity with potassium nitrate-based desensitizing agents. A bibliographic survey of the period from 2003 to 2019 was carried out in the PubMed and Scielo electronic databases. The Portuguese keywords "Desmineralização" "Hipersensibilidade da Dentina" and "Nitrato de Potássio" and the corresponding English words, "Demineralization" "Dentin Sensitivity" and "Potassium Nitrate" were used. The literature pointed out that potassium nitrate acted by increasing the concentration of potassium ions in the internal extremity of the dentinal tubules at a level that was high enough to inactivate the nerve endings of the pulp. This caused depolarization of the nerve fibers, and was shown to have a faster and more lasting action when compared with other desensitizing agents. Toothpastes based on $\mathrm{KNO}_{3}$ used alone or combined with other components were shown to be effective for treating dentin hypersensitivity. Potassium nitrate was most frequently incorporated into toothpastes and mouthwashes, in which the dosage that produced the most satisfactory results for the treatment of dentin hypersensitivity was the association with brushing and rinsing, respectively, twice daily for two minutes. All desensitizing agents based on potassium nitrate analyzed in the present study were able to effectively treat dentin hypersensitivity. Keywords: Demineralization. Dentin Sensitivity. Potassium Nitrate. 


\section{INTRODUÇÃO}

Devido à crescente expectativa de manutenção dos dentes na cavidade bucal, a hipersensibilidade dentinária (HD) tende a se tornar uma queixa ainda mais comum na prática clínica. Essa desordem é um sintoma relatado em dentes vitais com áreas de dentina expostas ao ambiente oral devido à degradação do esmalte ou cemento (JAMES et al., 2017), sendo caracterizada por uma dor aguda, de curta duração, decorrente da exposição dentinária a estímulos térmicos, tátil, osmótico ou químico que não podem ser associados a qualquer outro defeito dentário ou patologia (SILVA et al., 2011). Ainda que apresente um caráter agudo, e sobretudo por não ser espontânea, mas sim estimulada, a hipersensibilidade pode vir a ser considerada uma condição de dor crônica (RÖSING et al., 2009) com sua prevalência variando a depender da população alvo, dos métodos utilizados para seu diagnóstico e da análise dos dados (MAHESUTI et al., 2014).

A literatura aborda três principais teorias sobre os mecanismos de hipersensibilidade dentinária entretanto, nem a teoria da inervação direta tampouco a teoria do receptor de odontoblastos são favorecidas atualmente (LOW et al., 2015). A teoria hidrodinâmica é atualmente a mais aceita para explicar esse fenômeno, proposta por Brannstrom e Astrom, em 1964, preconiza que um estímulo externo é capaz de movimentar o material fluido ou semifluido no interior dos túbulos dentinários, excitando as terminações nervosas pulpares livres e consequentemente, causando a dor (RÖSING et al., 2009; ZHUANG, 2011). A patogênese da HD está comumente associada às lesões com perda de estrutura dentária como erosão, abrasão e abfração, lesões sem perda de estrutura dental, ou seja, as recessões gengivais e como efeito adverso da técnica de clareamento (DANTAS et al., 2013; MARAN et al., 2018).

O seu diagnóstico deve ser apoiado numa boa anamnese, exame clínico, exames complementares radiológicos e percepção do paciente, fazendo diagnóstico diferencial com outras causas de odontalgia (SANTOS et al., 2010; DELFIM, 2015). É importante destacar que uma superfície dentinária recém exposta pode se mostrar mais sensível aos estímulos do que a dentina coberta por smearlayer ou cemento, assim como a quantidade e a largura dos túbulos expostos, bem como a presença de dentina terciaria podem interferir na intensidade da dor provocada por estímulos exógenos (RÖSING et al., 2009). Em alguns casos a sensibilidade dentinária pode regredir espontaneamente, quando isso não ocorre, existem inúmeros tipos de terapias para o seu tratamento (SANTOS et al., 2010). Entre as formas de tratamento indicadas, incluem-se terapias de ação hiperestésicas, vernizes cavitários, agentes com ação oclusiva sobre os túbulos dentinários, precipitação de proteínas, deposição de partículas, aplicação de películas impermeabilizadoras, procedimentos restauradores, aplicação de laser e despolarização de fibras nervosas (MESQUITA $e t$ al., 2009).

As mais diversas formas de tratar a hipersensibilidade dentinária visam a ou reduzir a excitabilidade das fibras nervosas dentro do órgão pulpar ou obstruir os túbulos dentinários abertos. Dentre os diversos agentes que têm sido utilizados como dessensibilizantes, aqueles que continham íons potássio se mostraram eficazes no combate dessa problemática (SHARMA et al., 2012; JAMES et al., 2017). O Nitrato de Potássio sozinho ou associado com outro tratamento é amplamente recomendado na prática clínica odontológica (JAMES et al., 2017). Seu uso não obstrui os túbulos dentinários e nem promove a redução da condutibilidade hidráulica da dentina, mas atua aumentando a concentração de íons potássio na extremidade interna dos túbulos em nível suficiente para inativar às terminações nervosas da polpa provocando a despolarização das fibras nervosas, impedindo, assim, a passagem do estímulo doloroso ao sistema nervoso central (KWON et al., 2015; MESQUITA et al., 2009).

Em face ao exposto, pode-se concluir que a hipersensibilidade é uma condição crescente na clínica diária e que mais estudos devem ser realizados a fim de estabelecer um tratamento eficaz e duradouro. Diante disso, esse estudo teve como objetivo principal realizar uma revisão da literatura sobre a eficácia de alguns tratamentos da hipersensibilidade dentinária utilizando agentes dessensibilizantes a base de nitrato de potássio. 


\section{METODOLOGIA}

Esse trabalho foi elaborado a partir de uma revisão simples da literatura com o intuito de identificar as principais características das publicações. Esse tipo de estudo proporciona conhecimento atualizado sobre determinado assunto e analisa se o mesmo pode ou não ser aplicado na prática (FELIX et al., 2013). Essa modalidade de pesquisa é norteada por seis fases distintas: identificação do tema e elaboração da questão de pesquisa para a revisão; estabelecimento da estratégia de busca na literatura; seleção de estudos com base nos critérios de inclusão; leitura crítica, avaliação e categorização do conteúdo; análise e interpretação dos resultados (FELIX et al., 2013; BRANDÃO et al., 2017).

A questão norteadora proposta para o estudo foi: O Nitrato de Potássio é realmente eficaz para o tratamento da hipersensibilidade dentária? O recorte temporal compreendeu o período de janeiro/2003 a janeiro/2019 onde buscou englobar um período significativo, no que diz respeito à representatividade e quantidade das publicações, visto que, estudos diversos têm mostrado que a hipersensibilidade é um problema comum à população adulta, apresentando como fator etiológico uma serie de combinações de fatores que vão desde a recessão gengival até algumas doenças sistêmicas e desordens de oclusão (AGUIAR et al., 2005).

Para identificar as publicações que compuseram a revisão deste estudo, realizou-se uma busca online, mediante levantamento na Scielo e no PubMed/Medline, por meio de estratégia de busca com base nos termos: "Desmineralização" "Hipersensibilidade da Dentina" e "Nitrato de Potássio" e as correspondentes em inglês, "Demineralization" "DentinSensitivity" e "PotassiumNitrate". Os critérios de inclusão definidos para selecionar os estudos foram: artigos em inglês e português, aqueles que se enquadram no enfoque do trabalho e os mais relevantes em termos de delineamento das informações desejadas. Foram excluídas cartas ao editor, índices incomuns, revisões, artigos que não apresentavam o texto completo e artigos que embora apresentassem os descritores selecionados, não abordaram diretamente a temática. Considerando as bases científicas analisadas, 6 referências relacionadas ao tema atenderam os critérios de seleção estabelecidos.

Para proporcionar a categorização dos estudos, com o intuito de análise, síntese dos artigos que atenderam aos critérios de inclusão e a compreensão das informações, um instrumento de coleta de dados foi elaborado pelos autores no Microsoft Word 2007 que contemplou os seguintes aspectos, considerados pertinentes: título do periódico, ano de publicação, autoria, título da pesquisa, base de dados ou biblioteca virtual, objetivo, métodos, resultados e conclusões do estudo e nível de evidência ( 1 - revisões sistemáticas ou metanálise de relevantes ensaios clínico; 2 evidências de pelo menos um ensaio clínico randomizado controlado bem delineado; 3 - ensaios clínicos bem delineados sem randomização; 4 - estudos de coorte e de caso-controle bem delineado; 5 - revisão sistemática de estudos descritivos e qualitativos; 6 - evidências de um único estudo descritivo ou qualitativo; 7 - opinião de autoridades ou comitês de especialistas incluindo interpretações de informações não baseadas em pesquisas)(URSI; GALVÃO, 2006; BRANDÃO $e t$ al., 2017).

Os dados obtidos a partir do instrumento de coleta estão apresentados por meio de quadros, de forma que possibilite um melhor entendimento dos estudos da revisão integrativa, e se encontram expostos de forma descritiva. Por meio da análise temática ou categorial, tipo de técnica de análise de conteúdo, operou-se de desmembramento do texto em categorias, segundo reagrupamentos sistemáticos analógicos (BRANDÃO et al., 2017). 
Assim como qualquer outra sensação dolorosa no corpo, a hipersensibilidade dentária passou a ser vista pela população como um importante problema de saúde, levando até a uma maior preocupação dos indivíduos com seu estado de saúde (RÖSING et al., 2009). Dessa forma, várias estratégias de tratamento surgiram visando a reduzir ou potencialmente eliminar essa problemática as quais podem ser divididas através do seu mecanismo de ação nas que resultam na oclusão física dos túbulos e nas que bloqueiam a transmissão neural nos tecidos pulpares e despolarizam quimicamente a sinapse nervosa (JAMES et al., 2017). Devido ao aumento da relevância dessa condição, vários estudos surgiram para avaliar a eficácia dos inúmeros agentes dessensibilizantes que utilizam diversos componentes ativos (Quadro 1) que variam desde fluoretos até tratamentos que empregam nanopartículas biomiméticas.

Quadro 1 - Distribuição dos estudos segundo código, periódico, ano, autoria, título, base de dados ou biblioteca virtual, objetivo e métodos

\begin{tabular}{|c|c|c|c|}
\hline $\begin{array}{l}\text { Periódicos } \\
\text { Autores / Ano } \\
\text { Cód. do Artigo }\end{array}$ & $\begin{array}{c}\text { Título } \\
\text { Base de dados }\end{array}$ & Objetivo & Métodos / Nível de evidência (NE) \\
\hline $\begin{array}{l}\text { Journal of Indian } \\
\text { Society of } \\
\text { Periodontology } \\
\text { Tevatia et al. } \\
\text { (2017) } \\
\text { A1 }\end{array}$ & $\begin{array}{l}\text { Comparative } \\
\text { clinical evaluation } \\
\text { of gallium- } \\
\text { aluminum-arsenide } \\
\text { diode laser and } \\
\text { potassium nitrate in } \\
\text { treating dentinal } \\
\text { hypersensitivity. } \\
\text { PUBMED }\end{array}$ & $\begin{array}{l}\text { Tratar a HSDC com } \\
\text { concentração química } \\
\text { mínima e menor nível } \\
\text { de energia do laser } \\
\text { com maior período de } \\
\text { acompanhamento. }\end{array}$ & $\begin{array}{l}\text { Cento e vinte pacientes foram divididos aleatoriamente em } \\
\text { quatro grupos: (i) Grupo } 1-5 \% \text { de nitrato de potássio (KNO3); } \\
\text { (ii) Grupo } 2 \text { - laser de diodo de gálio-arsenieto de alumínio } \\
\text { ( } 62,2 \mathrm{~J} / \mathrm{cm} 2 \text {, comprimento de onda - } 980 \mathrm{~nm} \text {, modo de pulso } \\
\text { sem contato e potência em watts - } 0,5 \mathrm{~W} \text { ); (iii) Grupo } 3 \text { - } \\
\text { KNO3 combinado a } 5 \% \text { e o laser de diodo; e (iv) Grupo } 4 \text { - } \\
\text { placebo (controle). As pontuações da escala visual analógica } \\
\text { (VAS) foram registradas, analisadas e comparadas a estímulos } \\
\text { táteis, água fria e testes de jato de ar em intervalos diferentes } \\
\text { por } 6 \text { semanas. (N.E.:2). }\end{array}$ \\
\hline $\begin{array}{l}\text { Chinese Journal } \\
\text { of Dental } \\
\text { Research } \\
\text { Mahesuti et al. } \\
\text { (2014) } \\
\text { A2 }\end{array}$ & $\begin{array}{l}\text { Short-term Efficacy } \\
\text { of Agents } \\
\text { Containing KNO3 } \\
\text { or CPP-ACP in } \\
\text { Treatment of Dentin } \\
\text { Hypersensitivity. } \\
\text { PUBMED }\end{array}$ & $\begin{array}{l}\text { Avaliar a eficácia a } \\
\text { curto prazo de agentes } \\
\text { contendo KNO3 ou } \\
\text { fosfopeptídeo de } \\
\text { caseína-fosfato de } \\
\text { cálcio amorfo (CPP- } \\
\text { ACP) no tratamento da } \\
\text { hipersensibilidade } \\
\text { dentinária. }\end{array}$ & $\begin{array}{l}\text { UltraEZ, contendo KNO3 e Pasta MI, contendo CPP-ACP } \\
\text { foram aplicados neste estudo. A hipersensibilidade dentinária } \\
\text { de } 102 \text { indivíduos foi estabelecida por um estímulo tátil com } \\
\text { uma sonda de pressão Yeaple, e o grau de hipersensibilidade } \\
\text { foi medido usando uma escala visual analógica (VAS). Os } \\
\text { pacientes foram divididos em quatro grupos: A, B, C e D, } \\
\text { utilizando uma tabela de números aleatórios. UltraEZ, um } \\
\text { placebo de UltraEZ, MI Paste e um placebo de MI Paste foram } \\
\text { aplicados ao grupo A, B, C e D, respectivamente, por } 2 \\
\text { semanas. A hipersensibilidade dentinária foi medida usando } \\
\text { VAS antes do tratamento (linha de base), nos dias } 2,7 \text { e } 14 \\
\text { durante o tratamento e nos dias } 30 \text { e } 60 \text { pós-tratamento. } \\
\text { (N.E.:2). }\end{array}$ \\
\hline $\begin{array}{l}\text { Revista Dor } \\
\text { Cavalcante et al. } \\
\text { (2015) } \\
\text { A3 }\end{array}$ & $\begin{array}{l}\text { Improvement of } \\
\text { cervical dentin } \\
\text { hypersensitivity } \\
\text { after two different } \\
\text { treatments. } \\
\text { SCIELO }\end{array}$ & $\begin{array}{l}\text { Avaliar clinicamente a } \\
\text { eficácia de dois } \\
\text { tratamentos para } \\
\text { melhorar a } \\
\text { hipersensibilidade } \\
\text { dentinária cervical, } \\
\text { bem como a duração } \\
\text { de seus efeitos. }\end{array}$ & $\begin{array}{l}\text { O estudo foi desenvolvido como um estudo clínico, duplo- } \\
\text { cego e comparativo onde foram selecionados } 14 \text { pacientes com } \\
\text { hipersensibilidade dentinária cervical atendidos na Clínica } \\
\text { Faculdade de Odontologia do Centro Universitário Cesmac. } \\
\text { Os participantes foram divididos em dois grupos de sete } \\
\text { indivíduos. O primeiro grupo foi submetido a tratamento com } \\
\text { dessensibilizante com nitrato de potássio a } 5 \% \text { (Nano P®- } \\
\text { FGM) e o segundo grupo recebeu aplicações de verniz } \\
\text { fluoretado (Fluorniz@-SS White). Ambos os tratamentos } \\
\text { seguiram as instruções dos fabricantes. (N.E.:2). }\end{array}$ \\
\hline $\begin{array}{l}\text { International } \\
\text { Journal of } \\
\text { Dental Hygiene } \\
\text { Katanec et al. } \\
\text { (2016) } \\
\text { A4 }\end{array}$ & $\begin{array}{l}\text { New toothpaste to } \\
\text { deal with dentine } \\
\text { hypersensitivity: } \\
\text { double-blind } \\
\text { randomized } \\
\text { controlled clinical } \\
\text { trial. } \\
\text { PUBMED }\end{array}$ & $\begin{array}{l}\text { Avaliar um efeito } \\
\text { combinado de } \\
\text { ingredientes ativos } \\
\text { contidos em um novo } \\
\text { creme dental na } \\
\text { redução da } \\
\text { hipersensibilidade } \\
\text { dentinária. }\end{array}$ & $\begin{array}{l}\text { O creme dental testado continha ingredientes ativos nitrato de } \\
\text { potássio }(5,00 \%) \text { e citrato de zinco }(0,50 \%) \text {. Dor ao estímulo } \\
\text { de água e sopro de ar foram relatados em dentes hipersensíveis } \\
\text { e medidos usando a EVA (Escala Visual Analógica). O grau } \\
\text { de dor ao estímulo de água foi detectado depois que cada } \\
\text { sujeito bebeu alguns goles de água que foi fervida até a } \\
\text { temperatura ambiente. Os dados foram coletados sobre a } \\
\text { história odontológica de cada sujeito. (N.E.:2). }\end{array}$ \\
\hline
\end{tabular}


Quadro 1, cont.

\begin{tabular}{|c|c|c|c|}
\hline $\begin{array}{l}\text { Shanghai } \\
\text { Journal of } \\
\text { Stomatology } \\
\text { Zhuang e Cao } \\
\text { (2011) }\end{array}$ & $\begin{array}{c}\text { Efficacy of a } \\
\text { dentifrice } \\
\text { containing } 5 \% \\
\text { potassium nitrate on } \\
\text { dental } \\
\text { hypersensitivity. }\end{array}$ & $\begin{array}{l}\text { Avaliar a eficácia de } \\
\text { um dentifrício } \\
\text { contendo } 5 \% \text { de } \\
\text { nitrato de potássio } \\
\text { (teste) na } \\
\text { hipersensibilidade } \\
\text { dentária em } \\
\text { comparação com o } \\
\text { dentifrício fluoretado } \\
\text { (controle) durante um } \\
\text { período de } 4 \text { semanas. }\end{array}$ & $\begin{array}{l}\text { O desenho do estudo foi um ensaio clínico randomizado, } \\
\text { duplo-cego e controlado. } 63 \text { indivíduos foram divididos } \\
\text { aleatoriamente em } 2 \text { grupos, com } 31 \text { receberam um dentifrício } \\
\text { de nitrato de potássio a } 5 \% \text { (grupo experimental) e os outros } \\
32 \text { receberam um dentifrício fluoretado (grupo controle). } \\
\text { Ambos os grupos foram instruídos a escovar os dentes duas } \\
\text { vezes ao dia. Um estímulo de jato de ar foi usado para medir o } \\
\text { grau de dor usando uma escala EVA (Escala Visual } \\
\text { Analógica). Os dados foram analisados pelo teste não } \\
\text { paramétrico Mann-Whitney U e teste de Wilcoxon com o } \\
\text { pacote de software SPSS13.0. (N.E.:2). }\end{array}$ \\
\hline $\begin{array}{l}\text { American } \\
\text { Journal of } \\
\text { Dentistry } \\
\text { Hall et al. (2017) } \\
\text { A6 }\end{array}$ & $\begin{array}{c}\text { Efficacy of an } \\
\text { experimental } 3 \% \\
\text { potassium nitrate } \\
\text { mouthwash in } \\
\text { providing long-term } \\
\text { relief from dentin } \\
\text { hypersensitivity: An } \\
\text { 8-week randomized } \\
\text { controlled study } \\
\text { (Study 1) } \\
\text { PUBMED }\end{array}$ & $\begin{array}{l}\text { Para avaliar a eficácia } \\
\text { de um enxaguatório } \\
\text { bucal experimental } \\
\text { contendo } 3 \% \text { de } \\
\text { nitrato de potássio } \\
\text { (KNO3) no alívio da } \\
\text { hipersensibilidade } \\
\text { dentinária quando } \\
\text { usado como adjuvante } \\
\text { da escovação com } \\
\text { creme dental com } \\
\text { flúor em comparação } \\
\text { com o uso do mesmo } \\
\text { creme dental sozinho. }\end{array}$ & $\begin{array}{l}\text { Este foi um de três estudos de } 8 \text { semanas, randomizados, com } \\
\text { dois tratamentos, cegos para examinadores, de desenho } \\
\text { paralelo, em um único local, em indivíduos saudáveis com } \\
\text { hipersensibilidade dentinária auto-relatada e diagnosticada } \\
\text { clinicamente. Os indivíduos foram randomizados para receber } \\
\text { creme dental com flúor mais KNO3 } 3 \% \text { ou o mesmo creme } \\
\text { dental com flúor sozinho, e instruídos a usar o tratamento } \\
\text { alocado duas vezes ao dia durante as } 8 \text { semanas seguintes. A } \\
\text { hipersensibilidade dentinária foi avaliada no início do estudo e } \\
\text { após } 4 \text { e } 8 \text { semanas de tratamento por meio da avaliação das } \\
\text { respostas aos estímulos evaporativos (ar) e táteis [medidos } \\
\text { pela Escala de Sensibilidade de Schiff, uma escala de } \\
\text { avaliação visual (VRS) e limiar tátil, respectivamente], e } \\
\text { usando o Questionário de Experiência de Hipersensibilidade } \\
\text { Dentina (DHEQ; um instrumento de qualidade de vida } \\
\text { validado para hipersensibilidade dentinária). (N.E.:2). }\end{array}$ \\
\hline
\end{tabular}

Fonte: os autores.

Clinicamente, a hipersensibilidade dentária caracteriza-se por uma dor aguda de curta duração, em resposta a um estímulo sobre a dentina exposta, normalmente térmico, evaporativo, tátil, osmótico ou químico, o qual não apresenta relação com qualquer outro defeito ou patologia dentária (SILVA; GINJEIRA, 2011). Terapias dessensibilizantes que atuam na transmissão neuronal de estímulos dolorosos e na resposta inflamatória pulpar são de grande importância clínica já que se acredita que esses agentes reduzem a excitabilidade do nervo intradental (PAULA et al., 2018). O Nitrato de Potássio $\left(\mathrm{KNO}_{3}\right)$ pode ser utilizado na prática clínica de maneira isolada, associado a outros agentes e como coadjuvantes de outro tratamento (HALL et al., 2017), sendo usado em diferentes concentrações e viscosidades (KATANEC et al., 2016), pois apresenta um efeito calmante sobre o nervo, evitando a repolarização subsequente após a despolarização, obstruindo assim o ciclo da dor (PAULA et al., 2018).

Os dentrifícios são os meios mais usualmente utilizados no tratamento da HD. Isso se explica devido ao seu baixo custo, facilidade de aplicação, por não serem invasivos e porque podem ser utilizados pelo paciente em casa (SBRUZZI, 2017). Katanec et al. (2016) compararam a eficácia de um creme dental impregnado de 5\% de nitrato de potássio como agente dessensibilizante, obtendo uma melhora de $29 \%$ nas duas primeiras semanas de uso e $40 \%$ após quatro semanas. Também Zhuang e Cao (2011), avaliaram um dentríficio contendo 5\% de nitrato de potássio (teste) comparado a um dentrifício fluoretado (controle) através da escala visual analógica (EVA), durante a quarta semana de uso, onde observaram queos pacientes do grupo teste obtiveram redução significativa na EVA quando comparados ao grupo controle (Quadro 2). Concluindo que o nitrato de potássio possui ação dessensibilizante quando incorporado a dentríficios.

Outra abordagem do nitrato de potássio como agente dessensibilizante de forma caseira é incorporando-o a enxaguatórios (SBRUZZI, 2017). Hall et al. (2017) avaliou a eficácia de um enxaguatório bucal experimental com $\mathrm{KNO}_{3} 3 \%$ no alívio da hipersensibilidade dentinária quando usado como coadjuvante à escovação com creme dental fluoretado comparado ao uso do mesmo creme dental sozinho, vide Quadro 1, obtendo que o grupo dentifrício mais colutório apresentou maiores reduções na sensibilidade em ambos os momentos para todas as medidas clínicas como 
pode-se observar na Quadro 2. Assim, evidencia-se outra possibilidade de aplicação deste agente dessensibilizante, contudo é importante salientar que pacientes que já possuem HD devem associar o uso do colutório a cremes dentais com flúor e não abrasivos (SBRUZZI, 2017).

Uma nova perspectiva proposta para o tratamento da HD é associação de técnicas ou agentes dessensibilizantes. Tevatia et al. (2017) realizaram uma avaliação clínica comparativa do laser diodo de arsenieto de gálio-alumínio associado ao nitrato de potássio no tratamento da hipersensibilidade dentinária (Quadro 1), o uso sinérgico de $5 \%$ de $\mathrm{KNO}_{3}$ e laser diodo reduziu significativamente a dor que se mostrou quase insignificante após a $6^{\mathrm{a}}$ semana do estudo (97\% $99 \%$ da dor foi relatada como aliviada). Essa associação apresenta como resultado a ação neural através do nitrato de potássio e a indução de alterações na rede de transmissão nervosa dentro da polpa que produz dentina secundária neoformada e consequente obliteração fisiológica dos canalículos dentinários através do laser, obtendo assim, resultados promissores na melhora da dor e da qualidade de vida dos pacientes (Quadro 2).

Quadro 2 - Código do artigo, categoria temática e síntese dos resultados e conclusões

\begin{tabular}{|c|c|c|}
\hline $\mathrm{CA}$ & Resultados & Conclusão \\
\hline$A 1$ & $\begin{array}{l}\text { O uso sinérgico de KNO3 5\% e laser de diodo (Grupo 3) reduziu } \\
\text { significativamente a dor de HSDC, que era quase insignificante após a } 6^{a} \\
\text { semana }(97 \%-99 \% \text { da dor foi relatada como aliviada) e mostrou resultados } \\
\text { mais promissores do que quaisquer outros grupos estudados. Além disso, o } \\
\text { laser de diodo (Grupo } 2) \text { mostrou resultados melhores do que } 5 \% \text { KNO3 } \\
\text { (Grupo 1). ANOVA unilateral e teste post hoc de correção de Bonferroni } \\
\text { revelaram a combinação de grupos com diferenças significativas nas } \\
\text { pontuações médias de EVA nos diferentes intervalos de tempo }(\mathrm{P}<0,01) \text {. }\end{array}$ & $\begin{array}{l}\text { Convencionalmente, a aplicação } \\
\text { combinada de KNO3 } 5 \% \text { com o laser de } \\
\text { diodo pode ser recomendada para o } \\
\text { tratamento de pacientes com HSDC. }\end{array}$ \\
\hline$A 2$ & $\begin{array}{l}\text { A eficácia do UltraEZ na hipersensibilidade dentinária foi } \\
\text { significativamente melhor do que a do grupo placebo correspondente no } \\
\text { dia } 7 \text { durante o tratamento, enquanto a eficácia da pasta MI exibiu melhor } \\
\text { do que a do grupo placebo no dia } 14 \text { durante o tratamento. No entanto, não } \\
\text { houve diferenças entre a eficácia dos dois agentes no dia } 14 \text { durante o } \\
\text { tratamento, no dia } 30 \text { ou no dia } 60 \text { após o tratamento. }\end{array}$ & $\begin{array}{l}\text { Ambos UltraEZ e MI Paste tiveram um } \\
\text { efeito significativo na hipersensibilidade } \\
\text { dentinária. UltraEZ mostrou efeitos mais } \\
\text { rápidos do que a pasta MI, mas a pasta } \\
\text { MI teve uma ação sustentada maior após } \\
\text { o tratamento do que a UltraEZ. }\end{array}$ \\
\hline$A 3$ & $\begin{array}{l}\text { As avaliações clínicas foram realizadas } 7,14,21 \text { dias e } 1 \text { mês após o } \\
\text { tratamento. Após a análise estatística dos resultados, foi possível concluir } \\
\text { que os produtos melhoraram a hipersensibilidade dentinária cervical inicial } \\
\text { nos elementos dentários estudados após suas aplicações e que o efeito } \\
\text { dessensibilizante do nitrato de potássio foi mais duradouro em relação ao } \\
\text { verniz fluoretado. }\end{array}$ & $\begin{array}{l}\text { O efeito dessensibilizante do nitrato de } \\
\text { potássio foi mais duradouro em } \\
\text { comparação com o verniz fluoretado. }\end{array}$ \\
\hline$A 4$ & $\begin{array}{l}\text { Uma redução significativa na hipersensibilidade dentinária (26-29\% após } 2 \\
\text { semanas versus } 40 \% \text { após } 4 \text { semanas) foi detectada no grupo testado. Os } \\
\text { controles também mostraram redução significativa para ambos os } \\
\text { estímulos, mas em menor taxa. Uma diferença estatisticamente } \\
\text { significativa para sopro de ar e estímulos de água mostrou o maior impacto } \\
\text { para o grupo testado após } 4 \text { semanas }(\mathrm{P}=0,033, \mathrm{P}=0,006) \text {. }\end{array}$ & $\begin{array}{l}\text { Este estudo apoia a eficácia do creme } \\
\text { dental testado na redução do fenômeno de } \\
\text { hipersensibilidade dentinária após } 4 \\
\text { semanas de uso. }\end{array}$ \\
\hline$A 5$ & $\begin{array}{l}\text { A EVA (linha de base }=68,29 \pm 8,263,4 \text { semanas }=31,71 \pm 6,378 \text { ) foi } \\
\text { significativamente reduzido ao longo do período de } 4 \text { semanas no grupo } \\
\text { experimental }(\mathrm{P}<0,05) \text {. Após } 4 \text { semanas, a EVA no grupo experimental foi } \\
\text { significativamente menor do que no grupo controle }(\mathrm{P}<0,05) \text {. }\end{array}$ & $\begin{array}{l}\text { O estudo demonstra que o dentifrício } \\
\text { contendo } 5 \% \text { de nitrato de potássio reduz } \\
\text { efetivamente a hipersensibilidade } \\
\text { dentária. }\end{array}$ \\
\hline A6 & $\begin{array}{l}\text { Um total de } 216 \text { indivíduos foram randomizados e } 214 \text { completaram o } \\
\text { estudo. Ambos os grupos de tratamento demonstraram reduções } \\
\text { estatisticamente significativas da linha de base para cada medida clínica de } \\
\text { sensibilidade }(\mathrm{P} \leq 0,01) \text { nas semanas } 4 \text { e } 8 \text {. O uso de colutório KNO3 a } 3 \% \\
\text { após escovar com creme dental com flúor resultou em reduções } \\
\text { estatisticamente significativamente maiores na sensibilidade a um estímulo } \\
\text { evaporativo (ar) (pontuação média de Schiff e VRS médio, P <0,001; } \\
\text { pontuação média de Schiff do objetivo primário na semana } 8, \mathrm{P}<0,0001) \text { e } \\
\text { limiar tátil estatisticamente significativamente maior (P <0,001) nas } \\
\text { semanas } 4 \text { e } 8 \text { em comparação com o creme dental sozinho. As respostas } \\
\text { do DHEQ refletiram os resultados clínicos para vários parâmetros, } \\
\text { indicando uma melhoria significativa na qualidade de vida relacionada à } \\
\text { saúde bucal após } 8 \text { semanas de uso do enxaguatório bucal KNO3 a 3\%. }\end{array}$ & $\begin{array}{l}\text { Os resultados deste estudo sugerem que o } \\
\text { uso diário de um anti-séptico bucal com } \\
\text { KNO3 a 3\% como adjuvante da } \\
\text { escovação com creme dental com flúor } \\
\text { fornece melhorias clinicamente } \\
\text { relevantes na hipersensibilidade } \\
\text { dentinária após } 8 \text { semanas de uso duas } \\
\text { vezes ao dia. }\end{array}$ \\
\hline
\end{tabular}

Nota: CA - Código do Artigo

Fonte: os autores. 
Na tentativa de aliviar ou amenizar o desconforto causado por essa patologia também foram testados outros agentes dessensibilizantes. Mahesuti et al. (2014) avaliaram a eficácia a curto prazo de agentes contendo $\mathrm{KNO}_{3}$ ou fosfopeptídeo de fosfato de cálcio amorfo (CPP-ACP) no tratamento da hipersensibilidade dentinária de cento e dois indivíduos como observa-se na Quadro 1. Os agentes utilizados nesse estudo foram o UltraEZ, contendo $\mathrm{KNO}_{3}$ e MI Paste, contendo CPP-ACP. $\mathrm{O}$ UltraEZ apresentou efeito mais rápido enquanto o MI Paste, mais duradouro. Acredita-se que isso se deva ao mecanismo de ação dos produtos testados já que o CPP-ACP tem ação físico-química, agindo em áreas desmineralizadas, as quais são extremamente reativas, induzindo a remineralização e/ou obliterando túbulos dentinários expostos enquanto o $\mathrm{KNO}_{3}$ tem ação neural, bloqueando a transmissão de estímulos nos tecidos pulpares, ou seja, agindo na sintomatologia da problemática e não na etiologia.

Já Cavalcante et al. (2015) compararam a eficácia de dois tratamentos para melhorar a hipersensibilidade dentinária cervical: dessensibilizante a base de $\mathrm{KNO}_{3}$ a $5 \%$ (Nano $\mathrm{P} 囚-\mathrm{FGM}$ ) e aplicação de verniz fluoretado (Fluorniz ${ }^{\circledR}-S S$ White), bem como a duração de seus efeitos, tendo com resultados que ambos eram eficazes no tratamento da $\mathrm{HD}$, mas o que continha $\mathrm{KNO}_{3}$ a $5 \%$ obteve resultados mais duradouros (Quadro 2).

De uma forma geral, há uma concordância entre os autores sobre a eficácia do nitrato de potássio no combate a hipersensibilidade dentinária. A sua concentração varia nos estudos entre $3 \mathrm{e}$ $5 \%$, sendo a de $5 \%$ a mais utilizada e se apresenta para uso tópico, em dentifrícios e colutórios. Quanto ao início de ação há uma unanimidade que o $\mathrm{KNO}_{3}$ possui resposta rápida e que após 4 semanas de uso há uma redução significativa da dor e melhora da qualidade de vida nos pacientes. No entanto, os estudos não debatem se há recidivas nos quadros de HD nos pacientes a longo prazo, o que ainda hoje é um grande desafio para odontologia.

\section{CONCLUSÃO}

$\mathrm{O}$ uso do $\mathrm{KNO}_{3}$ atua aumentando a concentração de íons potássio na extremidade interna dos túbulos em nível suficiente para inativar às terminações nervosas da polpa provocando a despolarização das fibras nervosas; quando comparamos seu efeito com produtos contendo outras substâncias como CPP-ACP e verniz fluoretado, o nitrato de potássio possui início de ação mais rápido que o $\mathrm{CPP}-\mathrm{ACP}$ e uma durabilidade maior de resultados que o verniz fluoretado.

Dentifrícios a base de $\mathrm{KNO}_{3}$ sozinho ou combinados com outros componentes são capazes de tratar de maneira eficaz a hipersensibilidade dentinária. As formas de incorporação do nitrato de potássio mais frequentes são através de dentrifícios e colutórios bucais onde a posologia que mais apresentou resultados satisfatórios para o uso dos mesmos no tratamento da HD foi duas vezes ao dia durante dois minutos.

\section{REFERÊNCIAS}

AGUIAR, F. H. B. et al. Hipersensibilidade Dentinária - causas e tratamento. Uma revisão da literatura. Revista do Instituto de Ciências da Saúde, v. 23, n. 1, p. 67-71, 2005.

BRANDÃO, B. M. G. M. et al. Representações sociais da equipe de enfermagem perante o paciente com HIV/AIDS: uma revisão integrativa. Revista de Enfermagem UFPE online, v. 11, n. 2, p. 625-633, 2017.

BRITO, L. P. P. Avaliação de Superfícies Dentinárias Cervicais Tratadas com Agentes Dessensibilizantes: Estudo por Meio de Microscopia Eletrônica de Varredura. [Monografia] Universidade Federal do Pará, Pará, 2005. 
CAVALCANTE, M. S. et al. Improvement of cervical dentin hypersensitivity after two different treatments. Revista Dor, v. 16, n. 4, p. 259-262, 2015.

DANTAS, E. M. et al. Tratamento da hipersensibilidade dentinária cervical com laser de baixa potência - revisão de literatura. Revista Odontologia Clínico-Científica do CRO-PE, v. 12, n. 1, p. 7-11, 2013.

DELFIM, P. C. R. M. Hipersensibilidade Dentinária: Estudo clínico piloto. 2015. $58 \mathrm{f}$. Dissertação (Mestrado Integrado em Medicina Dentária) - Universidade de Lisboa, Lisboa, 2015.

FELIX, Z. C. et al.Eutanásia, distanásia e ortotanásia: revisão integrativa da literatura. Ciência \& Saúde Coletiva, v. 18, n. 9, p. 2733-2746, 2013.

HALL, C. et al. Efficacy of an experimental 3\% potassium nitrate mouthwash in providing longterm relief from dentin hypersensitivity:an 8-week randomized controlled study (study 1). American Journal of Dentistry, v. 30, n. 1, p. 27-34, 2017.

HOEPPNER, M. G.; MASSAROLLO, S.; BREMM, L. L. Considerações clínicas das lesões cervicais não cariosas. Publication UEPG Ciências Biológicas e da Saúde, v. 13, n. 3/4, p. 81-86, 2007.

JAMES, J. M.; PURANIK, M. P.; SOWMYA, K. R. Dentinal Tubule Occluding Effect of Potassium Nitrate in Varied Forms, Frequencies and Duration: An In vitro SEM Analysis. Journal of Clinical and Diagnostic Research, v. 11, n. 8, p. ZC06-ZC08, 2017.

KATANEC, T. et al. New toothpaste to deal with dentine hypersensitivity: double-blind randomized controlled clinical trial. International Journal of Dental Hygiene, v. 16, n. 1, p. 7884, 2018.

KWON, S. R. et al. Spectrophotometric Evaluation of Potassium Nitrate Penetration into the Pulp Cavity. Operative Dentistry, v. 40, n. 6, p. 614-621, 2015.

LOW, S. B.; ALLEN, E. P.; KONTOGIORGOS, E. D. Reduction in dental hypersensitivity with nano-hydroxyapatite, potassium nitrate, sodium monoflurophosphate and antioxidants. The Open Dentistry Journal, n. 9, p. 92-97, 2015.

MAHESUTI, A. et al. Short-term efficacy of agents containing $\mathrm{KNO}_{3}$ or CPP-ACP in treatment of dentin hypersensitivity. Chinese Journal of Dental Research, v. 17, n. 1, p. 43-47, 2014.

MESQUITA C. R. et al. Hiperestesia dentinária: opções de tratamento. Revista Dentística online, v. 8, n. 18, p. 30-34, 2009.

PAULA, B. et al. Effect of photobiomodulation with low-level laser therapy combined with potassium nitrate on controlling post-bleaching tooth sensitivity: clinical, randomized, controlled, double-blind, and split-mouth study. Clinical Oral Investigations, v. 23, n. 6, p. 2723-2732, 2019.

RÖSING, C. K. et al. Dentine hipersensitivity: analysis of self-care products. Brazilian Oral Research, v. 23, n. 1, p. 56-63, 2009.

SANTOS, A. P. M. et al. Um sintoma preocupante: a hipersensibilidade dentinária. Revista Brasileira de Odontologia, v. 67, n. 2, p. 242-246, 2010. 
SBRUZZI, M. M. Agentes dessensibilizantes Nitrato De Potássio, Cloreto De Estrôncio E Fluoreto De Sódio: Revisão De Literatura. 2017. 37f. Trabalho de Conclusão de Curso (Bacharelado em Odontologia) - Universidade de Santa Cruz do Sul, Santa Cruz do Sul, 2017.

SHARMA, S.; SHETTY, N. J.; UPPOOR, A. Evaluation of the clinical efficacy of potassium nitrate desensitizing mouthwash and a toothpaste in the treatment of dentinal hypersensitivity. Journal of Clinical and Experimental Dentistry, v. 4, n. 1, p. e28-e33, 2012.

SILVA, B. S. et al. Ocorrência da hipersensibilidade dentinária e seus fatores de risco. Revista de Cirurgia e Traumatologia Buco-maxilo-facial, v. 11, n. 1, p. 9-12, 2011.

SILVA, M. F.; GINJEIRA, A. Hipersensibilidade dentinária: etiologia e prevenção. Revista Portuguesa de Estomatologia, Medicina Dentária e Cirurgia Maxilofacial, v. 52, n. 4, p. 217 224, 2011.

TEVATIA, S. et al. Comparative clinical evaluation of gallium-aluminum-arsenide diode laser and potassium nitrate in treating dentinal hypersensitivity. Journal of Indian Society of Periodontology, v. 21, n. 5, p. 391-397, 2017.

URSI, E. S.; GALVÃO, C. M. Prevenção de lesões de pele no perioperatório: revisão integrativa da literatura. Revista Latino-Americana de Enfermagem, v. 14, n. 1, p. 124-31, 2006.

ZHUANG, W. J.; CAO D. Efficacy of a dentifrice containing 5\% potassium nitrate on dental hypersensitivity. Shanghai Kou Qiang Yi Xue, v. 20, n. 6, p. 638-640, 2011. 